\title{
Research on the Seismic Strain Field Before Earthquake Above Ms7, CHINESE Mainland
}

\author{
Guofu Luo, Zhongwang Liu, Fenghe Ding ${ }^{*}$, Heqing Ma, Mingzhi Yang
}

Earthquake Administration of Ningxia Hui Autonomous Region, Yinchuan, China

Email address:

Luoguofu_05@163.com (Fenghe Ding)

*Corresponding author

\section{To cite this article:}

Guofu Luo, Zhongwang Liu, Fenghe Ding, Heqing Ma, Mingzhi Yang. Research on the Seismic Strain Field Before Earthquake Above Ms7, CHINESE Mainland. Earth Sciences. Vol. 8, No. 1, 2019, pp. 10-19. doi: 10.11648/j.earth.20190801.12

Received: December 12, 2018; Accepted: December 27, 2018; Published: January 30, 2019

\begin{abstract}
In the paper, seismic strain field is considered as a variable of seismic activity. The strain field is calculated before the earthquake above Ms7 in mainland China since 1980, and the abnormal change of time factor is extracted before the earthquake, by means of the natural orthogonal function expansion method. The results show that the time factor before the large earthquake will change suddenly, jump or jump down on stationary background. At least 3 typical fields will appear abnormal changes in the first 4 ones of seismic strain field before the earthquake, having the characteristics of multi-component display anomalies. The earliest time of abnormal occurrence is about 3 years before the earthquake. Moreover, there are some short-term time factor anomalies before some earthquakes. Through case studies the results of case studies show that the time factor anomaly extracted by strain field is more unique than seismic energy field in analyzing the anomaly of seismic activity. The difference between strain field and energy field is also discussed.
\end{abstract}

Keywords: Chinese Mainland, Seismic Strain Field, Seismic Energy Field, Natural Orthogonal Function Expansion Method, Time Factor

\section{Introduction}

It is well known that seismic activity is a random natural phenomenon. The purpose of seismicity research is to reveal the regularity of seismic activity, to explore the relationship between seismic anomalies and large earthquakes, and to seek solutions to earthquake forecast and prediction. Since earthquake prediction is an extremely complicated and difficult task, seismologists have long been trying to find effective methods for earthquake prediction, which is still in the exploratory stage.

Assuming a physical variable is a variable or several independent variables of a function, when scientific observation, observation makes the function every time can take various forms of numerical or fail to know in advance, such a function is known as the random function, the corresponding physical variables known as the random function. The method of random function field has been widely used in meteorological research [1-2].

Yang [3] first applied the theory of natural orthogonal function to study the relationship between the time anomaly characteristics of the earthquake activity energy field in Ningxia region and the moderate earthquake in China. Luo [4], then, the author used this method to the seismicity in Yunnan region of China, related research and systematic analysis of space-time field before and after the 1975 earthquake anomaly and the corresponding relation of medium-strong earthquakes; Luo [5] used this method to calculate the energy field of seismic activity in the northwest Yunnan to the southern Yunnan region of China extracted spatio-temporal anomaly, and predicted the strong earthquake of the region in the next three years. Yang [6] by using this method, the study found that 2008 Wenchuan county, Sichuan province area before an earthquake of magnitude Ms8.0 earthquake activity field and medium-term anomaly characteristics of time and space, and Wenchuan earthquake have good corresponding relation; Ma [7] by using this method, such as analysis of Yushu county of Qinghai province in 2010 before the magnitude Ms7.1 earthquake epicenter surrounding areas and along the Yushu fault zone seismic energy field of the time change, also appears the first half of the anomalies of time and space, and the earthquake; Yang [8] by using this method, summarizes 
the Chinese mainland before 30 times the magnitude Ms6 earthquake near the epicenter of the earthquake activity field variation characteristics of time factor, the vast majority of earthquake cases has obvious time anomaly; Luo [9] by using this method, studies Minxian county of Gansu province in 2013 before the magnitude Ms6.6 earthquake epicenter regional seismic energy field of the space-time abnormity characteristics and analysis of the evolution of the space before the earthquake energy field and the corresponding relation of the magnitude Ms6.6 earthquake epicenter. Through the above study of earthquake cases in recent years found that the main application before the main characteristic of natural orthogonal function expansion value corresponding to the typical field of time factor will appear obvious abnormal changes of sudden rise or fall has good corresponding relation with the future earthquake, and the time factor anomalies before earthquakes are generally appear in 1 to 3 years, in most cases is less than 2 years, show the anomaly characteristics and medium-term earthquake prediction. Therefore, the author believes that the method of orthogonal function expansion of seismicity may be an earthquake prediction method worthy of further research.

Yang[3], such as the random field theory application in earthquake prediction, this was the first time in China, in the United States, Europe and Japan and other places of earthquake strain field research in the field of haven't seen a relevant method of paper work.

In this paper, using the method of natural orthogonal function expansion, in mainland China since 1980 more than magnitude Ms7 earthquake, calculation of strain before earthquake activity, to extract the relevant characteristics of the time factor sequence, the relations between the time factor variation characteristics and earthquake, explore the future earthquake occurrence regularity, the strain field and the field of natural orthogonal function expansion, the results were compared to discuss found its way to some superiority, the actual earthquake prediction for the future work to provide the corresponding seismic basis.

\section{Calculation Method and Principle}

The variables such as temperature and pressure in meteorological observation represent the physical field in a fairly spatial range around the weather station, and they are functions of spatial position. Yang [3] proposed the grid method to construct the random field: the airport: suppose a study area is $S$, according to the regional seismicity level and research purposes, select a time interval is $\Delta t$, the observation time is divided into $m$ periods, $t_{i}=\Delta t \times i(i=1,2, \cdots, m)$, and the study area is divided into $n$ equal area elements, $\Delta s=\Delta x \times \Delta y$, Its central coordinate is $\left(x_{j}, y_{j}\right)(j=1,2, \cdots, n)$.

The observed value of each grid in each time period is $S_{i j}$, Take it as the value of the random function of $\left(x_{i}, y_{i}, t_{j}\right)(i, j=1,2, \cdots, n)$ in time and space coordinates.

In this paper, $E$ means earthquake energy release, $\sqrt{E}$ denotes the square root of the seismic energy, and the square root of the seismic energy should be proportional to the earthquake, that is $\sqrt{E}=C \varepsilon$ ( $C$ is related to the seismic source parameters), partly reflects the source area of strain changes. After the study area is meshed, the strain field function is established by $S=\sum_{i} \sqrt{E_{i}}$, and the matrix form

$$
S=\left[\begin{array}{cccc}
S_{11} & S_{12} & \cdots & S_{1 n} \\
S_{21} & S_{22} & \cdots & S_{2 n} \\
\vdots & \vdots & \vdots & \vdots \\
S_{m 1} & S_{m 2} & \cdots & S_{m n}
\end{array}\right]
$$

In China, earthquakes of magnitude $M \mathrm{~s} 7$ or above are all intra-plate earthquakes, and the source correlation parameters are assumed to be a constant. Therefore, in the sense of studying the nature of large earthquakes, the field function $S=\sum_{i} \sqrt{E_{i}}$ can be called the seismic strain field. Where $S_{i j}(i=1,2, \cdots, m, j=1,2, \cdots n)$ is the observation value of $i$ grid and $\mathrm{j}$ time period respectively. The energy is calculated according to the formula $\log E=4.8+1.5 M$ ( $E$ is the joule for the energy unit, $\mathrm{M}$ for the magnitude). The natural orthogonal function of $\mathrm{S}$ was carried out, then the time factor and space anomaly were calculated, and the relationship between the characteristics of spatiotemporal anomaly and the major earthquake was analyzed.

The natural orthogonal function expansion is to break the matrix $S$ into the orthogonal space function $X$ and the orthogonal time function $T$, and then multiply the two, and then sum them.

$$
S_{i j}=\sum_{p=1}^{n} T_{i p} X_{p j}\left\{\begin{array}{l}
i=1,2, \cdots, m \\
j=1,2, \cdots, n
\end{array}\right.
$$

It satisfies the orthogonality and normalization conditions.

$$
\begin{gathered}
\sum_{j=1}^{n} x_{k j} x_{l j}=\left\{\begin{array}{cc}
0 & k \neq l \\
1 & k=l
\end{array}\right. \\
\sum_{i=1}^{m} T_{i k} T_{i l}= \begin{cases}0 & k \neq l \\
\lambda_{k} & k=l\end{cases}
\end{gathered}
$$

By solving the characteristic equation of the corresponding covariance matrix $R=S^{\prime} S$, that is

$$
\left[\begin{array}{cccc}
R_{11} & R_{12} & \cdots & R_{1 n} \\
R_{21} & R_{22} & \cdots & R_{2 n} \\
\cdots & \cdots & \cdots & \cdots \\
R_{n 1} & R_{n 2} & \cdots & R_{n n}
\end{array}\right]\left[\begin{array}{l}
x_{1} \\
x_{2} \\
\cdots \\
x_{n}
\end{array}\right]=\lambda\left[\begin{array}{c}
x_{1} \\
x_{2} \\
\cdots \\
x_{n}
\end{array}\right]
$$

Get the eigenvector $\vec{x}_{k}$ and the eigenvalue $\lambda_{k}(k=1,2, \cdots, n)$. Its time factor (weight coefficient sequence) 
is expressed as:

$$
\vec{T}_{k}=S \vec{x}_{k} \quad k=1,2, \cdots, n
$$

The eigenvector $\vec{x}_{k}$ represents the spatial distribution of the different seismic strain fields, while the time factor $\vec{T}_{k}$ represents the time change of the strain field, which reflects the dynamic characteristics of different time strain fields.

Because the covariance matrix $\vec{R}$ is a real symmetric matrix, $n$ eigenvalues $\lambda_{k}$ are positive real Numbers. The eigenvalues are arranged by size, and the eigenvectors corresponding to the first few eigenvalues are called the main strain fields. Due to the rapid convergence of natural orthogonal functions, only a few eigenvectors (strain fields) can be used to match the total field with satisfactory precision. The changes of several main strain fields represent the spatiotemporal characteristics of the regional strain field. Assuming that the sum of all $n$ eigenvalues is $b_{0}$, the accuracy of the general field fitting for the former $l$ is $r_{l}$.

$$
r_{l}=\sum_{p=1}^{l} \lambda_{p} / b_{0}
$$

In the formula, $\lambda_{p}$ represents the $p$ th characteristic root.

Natural orthogonal function on the sum of each component of the earthquake and strain, but the $\mathrm{n}$ linear combination are not equally important, the biggest characteristic value of strain field occupies a large proportion in the total field, has a larger variable ratio (variance). Therefore, if the main variation characteristics of the strain field are described, it is not necessary to use all the expanded items, but only with the corresponding combination of the largest eigenvalues. This is equivalent to concentrating the main information of the original strain field on the first few main strain fields. Thus, the main strain field with abnormal change was found out, and the strain field with small degree of correlation with the large earthquake was excluded.

\section{Seismic data and Calculations}

\subsection{Seismic Data}

In this article, the use of seismic data is provided by the China earthquake networks center of national official weak earthquake catalogue, the different research area and the time period in the earthquake data are $b$ value, choosing a complete minimum each research area of earthquake magnitude lower limit (table 1); Considering the probability smaller medium-strong earthquakes, the earthquake had a greater influence on the strain of strain field in the study area, the earthquake has does not belong to the area to normal active, so the magnitude limit take $M_{\mathrm{L}} 5.4$ (converted to magnitude $M$ s5.0), and $\mathrm{K}-\mathrm{K}$ method to remove the aftershocks of the magnitude $M \mathrm{~s} 5.0$ earthquake. Different earthquake study area according to 0.5 arc multiplied by 0.5 arc square grid, in regional grid, and mainly adopts time sliding algorithm, and the time interval in 3 months, sliding step take 1 month, discretization of data processing. According to the formula (1) in the above research method, the seismic strain release matrix $\mathrm{S}$ is constructed, and then the natural orthogonal function is analyzed. By solving the covariance matrix R, the main field corresponding to the eigenvalue and eigenvalue of the strain field is obtained, and the time factor corresponding to the main field is obtained, and the spatial contour distribution in different research areas is obtained.

In this paper, the natural orthogonal function is used to analyze the regional strain field in the pre-earthquake $M \mathrm{~s} 7$ earthquake in China since 1980. As a result of the China earthquake networks monitoring capability is not uniform, especially in parts of Qinghai province and Tibet province network observation ability is low, and so on November 8, 1997 Tibetan Mani Ms7.5 earthquake and on November 14, 2001, Kunlun mountain west Ms8.1 earthquake due to the observation data is not yet complete, can't strain field calculation. In this paper, the data is recorded in the Chinese mainland, and the results are shown in table 1. The time factor change curve is shown in figure 1.

\begin{tabular}{|c|c|c|c|c|c|c|c|c|}
\hline No. & Earthquakedate & $\begin{array}{l}\text { Earthquake } \\
\text { location }\end{array}$ & $\begin{array}{l}\text { Earthquake } \\
\text { magnitude }(M s)\end{array}$ & $\begin{array}{l}\text { Research area } \\
\text { (Longitude/E, Latitude/N) }\end{array}$ & $\begin{array}{l}\text { Data } \\
\text { period }\end{array}$ & $\begin{array}{l}\text { Magnitude } \\
\operatorname{range}\left(M_{\mathrm{L}}\right)\end{array}$ & $\begin{array}{l}\text { Calculate the first } \\
4 \text { eigenvalues }\end{array}$ & $\begin{array}{l}\text { Calculate } \\
\text { accuracy }\end{array}$ \\
\hline 1 & Aug. 23, 1985 & $\begin{array}{l}\text { Xinjiang } \\
\text { Wuqia }\end{array}$ & 7.1 & $\begin{array}{l}\mathrm{N} 39.0 \text { to } 41.5 \\
\text { E74.5 to } 77.5\end{array}$ & $\begin{array}{l}\text { Jan., } 1975 \\
\text { to Jul., } \\
1985\end{array}$ & 2.8 to 5.4 & $\begin{array}{l}3.5912 \times 10^{3} \\
0.9524 \times 10^{3} \\
0.6985 \times 10^{3} \\
0.6403 \times 10^{3}\end{array}$ & 0.8329 \\
\hline 2 & Nov.16, 1988 & $\begin{array}{l}\text { Yunnan } \\
\text { lancang }\end{array}$ & 7.6 & $\mathrm{~N} 21.5$ to 23.5 , E99.0 to 101.0 & $\begin{array}{l}\text { Jan., } 1975 \\
\text { to Oct., } \\
1988\end{array}$ & 2.7 to 5.4 & $\begin{array}{l}235.8914 \\
155.9525 \\
136.1460 \\
99.0805\end{array}$ & 0.6800 \\
\hline 3 & Apr.2, 1990 & $\begin{array}{l}\text { Qinghai } \\
\text { Gonghe }\end{array}$ & 7.0 & N34.0 to 37.0, E98.5 to 101.5 & $\begin{array}{l}\text { Jan., } 1980 \\
\text { to Mar., } \\
1990\end{array}$ & 2.8 to 5.4 & $\begin{array}{l}91.0688 \\
58.5975 \\
40.8794 \\
15.9304\end{array}$ & 0.9227 \\
\hline 4 & Jun. 14, 1990 & $\begin{array}{l}\text { Xinjiang } \\
\text { Jimuna }\end{array}$ & 7.2 & $\mathrm{~N} 46.0$ to $49.0, \mathrm{E} 83.5$ to 86.5 & $\begin{array}{l}\text { Jan., } 1980 \\
\text { to May., } \\
1990\end{array}$ & 3.0 to 5.4 & $\begin{array}{l}13.1051 \\
10.3549 \\
3.6816 \\
1.5037\end{array}$ & 0.8021 \\
\hline 5 & Jul. 12, 1995 & China & 7.3 & $\mathrm{~N} 21.0$ to 24.0 & Jan., 1985 & 3.0 to 5.4 & $1.0670 \times 10^{3}$ & 0.8107 \\
\hline
\end{tabular}

Table 1. Parameters table of ten earthquakes above Ms7. 


\begin{tabular}{|c|c|c|c|c|c|c|c|c|}
\hline No. & Earthquakedate & $\begin{array}{l}\text { Earthquake } \\
\text { location }\end{array}$ & $\begin{array}{l}\text { Earthquake } \\
\text { magnitude }(M s)\end{array}$ & $\begin{array}{l}\text { Research area } \\
\text { (Longitude/E, Latitude/N) }\end{array}$ & $\begin{array}{l}\text { Data } \\
\text { period }\end{array}$ & $\begin{array}{l}\text { Magnitude } \\
\operatorname{range}\left(M_{\mathrm{L}}\right)\end{array}$ & $\begin{array}{l}\text { Calculate the first } \\
4 \text { eigenvalues }\end{array}$ & $\begin{array}{l}\text { Calculate } \\
\text { accuracy }\end{array}$ \\
\hline & & $\begin{array}{l}\text { borders } \\
\text { myanmar }\end{array}$ & & E98.5 to 102.0 & $\begin{array}{l}\text { to Jun., } \\
1995\end{array}$ & & $\begin{array}{l}0.5072 \times 10^{3} \\
0.2105 \times 10^{3} \\
0.1965 \times 10^{3}\end{array}$ & \\
\hline 6 & Feb. 3, 1996 & $\begin{array}{l}\text { Yunnan } \\
\text { Lijiang }\end{array}$ & 7.0 & $\begin{array}{l}\mathrm{N} 26.5 \text { to } 29.0 \\
\text { E98.4 to } 100.9\end{array}$ & $\begin{array}{l}\text { Jan., } 1985 \\
\text { to Jan. } \\
1996\end{array}$ & 2.8 to 5.4 & $\begin{array}{l}41.3727 \\
16.8231 \\
12.9331 \\
6.0894\end{array}$ & 0.7857 \\
\hline 7 & May. 12, 2008 & $\begin{array}{l}\text { Sichuan } \\
\text { Wenchuan }\end{array}$ & 8.0 & $\begin{array}{l}\text { N30.0 to } 33.5, \\
\text { E101.5 to } 106\end{array}$ & $\begin{array}{l}\text { Jan., } 1995 \\
\text { to Apr., } \\
2008\end{array}$ & 2.7 to 5.4 & $\begin{array}{l}647.9299 \\
213.4451 \\
157.4164 \\
96.7784\end{array}$ & 0.8585 \\
\hline 8 & Apr. 14, 2010 & $\begin{array}{l}\text { Qinghai } \\
\text { Yushu }\end{array}$ & 7.1 & $\begin{array}{l}\mathrm{N} 32.0 \text { to } 34.5, \\
\text { E95.5 to } 98.5\end{array}$ & $\begin{array}{l}\text { Jan., } 1995 \\
\text { to Apr., } \\
2010\end{array}$ & 3.0 to 5.4 & $\begin{array}{l}635.2714 \\
101.7391 \\
88.7516 \\
71.5995\end{array}$ & 0.9290 \\
\hline 9 & Apr. 20, 2013 & $\begin{array}{l}\text { Sichuan } \\
\text { Lushan }\end{array}$ & 7.0 & $\begin{array}{l}\mathrm{N} 29.5 \text { to } 31.5 \\
\text { E102.5 to } 105.5\end{array}$ & $\begin{array}{l}\text { Jan., } 2000 \\
\text { to Mar., } \\
2013\end{array}$ & 2.7 to 5.4 & $\begin{array}{l}298.9720 \\
259.1229 \\
33.7822 \\
26.0381\end{array}$ & 0.9178 \\
\hline 10 & Aug. 8, 2017 & $\begin{array}{l}\text { Sichuan } \\
\text { Jiuzaigou }\end{array}$ & 7.0 & $\begin{array}{l}\mathrm{N} 32.0 \text { to } 34.0 \\
\text { E103.0 to } 105.0\end{array}$ & $\begin{array}{l}\text { Jan., } 2000 \\
\text { to Jul., } \\
2017\end{array}$ & 2.7 to 5.4 & $\begin{array}{l}37.8619 \\
35.0843 \\
31.3636 \\
26.7762\end{array}$ & 0.6698 \\
\hline
\end{tabular}

Note: the time of earthquake in table 1 is Beijing time of China

Table 2. The parameters of the seismic strain field of 10 times Ms 7.0 or above were calculated. above Ms 7 .

\begin{tabular}{|c|c|c|c|c|c|c|c|c|}
\hline $\begin{array}{l}\text { No. } \\
\mathbf{r}\end{array}$ & $\begin{array}{l}\text { Earthquake } \\
\text { date }\end{array}$ & $\begin{array}{l}\text { Earthquak } \\
\text { e location }\end{array}$ & $\begin{array}{l}\text { Earthquake } \\
\text { magnitude (Ms) }\end{array}$ & $\begin{array}{l}\text { The first } \mathbf{k} \\
\text { time factor } T_{k}\end{array}$ & $\begin{array}{l}\text { Abnormal occurrence } \\
\text { time }\end{array}$ & $\begin{array}{l}\text { Maximum } \\
\text { anomalous } \\
\text { amplitude } \Delta T\end{array}$ & $\begin{array}{l}\text { The time } \\
\text { factor Mean } \\
\text { square error }\end{array}$ & $\begin{array}{l}\text { abnormal } \\
\text { strain field } \\
\text { ratio }\end{array}$ \\
\hline \multirow{4}{*}{1} & \multirow{4}{*}{ Aug. 23, 1985} & \multirow{4}{*}{$\begin{array}{l}\text { Xinjiang } \\
\text { Wuqia }\end{array}$} & \multirow{4}{*}{7.1} & first & Jan. to Feb., 1983 & -38.7708 & 5.3815 & 0.5085 \\
\hline & & & & second & Dec. 1982 to Feb. 1983 & 4.8120 & 2.7715 & 0.1349 \\
\hline & & & & third & Apr. to Jun., 1983 & -12.1061 & 2.3735 & 0.0989 \\
\hline & & & & fourth & Jun. to Aug., 1983 & 6.9730 & 2.2724 & 0.0907 \\
\hline \multirow{3}{*}{2} & \multirow{3}{*}{ Nov.16, 1988} & \multirow{3}{*}{$\begin{array}{l}\text { Yunnan } \\
\text { lancang }\end{array}$} & \multirow{3}{*}{7.6} & first & Oct. 1987 to Oct. 1988 & $-5.9957 \vee$ & 1.2030 & 0.2558 \\
\hline & & & & third & Aug. to Oct., 1988 & -1.1282 & 0.9139 & 0.1476 \\
\hline & & & & fourth & Sep. to Nov., 1987 & -5.6940 & 0.7797 & 0.1074 \\
\hline \multirow{4}{*}{3} & \multirow{4}{*}{$\begin{array}{l}\text { Apr.2, } \\
1990\end{array}$} & \multirow{4}{*}{$\begin{array}{l}\text { Qinghai } \\
\text { Gonghe }\end{array}$} & \multirow{4}{*}{7.0} & first & Aug. 1987 to Apr. 1988 & -3.8884 & 0.8712 & 0.4070 \\
\hline & & & & second & Sep. 1987 to Jun. 1988 & 3.5326 & 0.6988 & 0.2618 \\
\hline & & & & third & Apr. to Jun., 1989 & -3.6195 & 0.5837 & 0.1827 \\
\hline & & & & 4 & Mar. to May, 1987 & 2.0295 & 0.3644 & 0.0712 \\
\hline \multirow{3}{*}{4} & \multirow{3}{*}{ Jun. 14, 1990} & \multirow{3}{*}{$\begin{array}{l}\text { Xinjiang } \\
\text { Jimuna }\end{array}$} & \multirow{3}{*}{7.2} & first & Sep. to Nov., 1989 & 1.9896 & 0.3277 & 0.3670 \\
\hline & & & & second & Jul. to Sep., 1989 & 1.9925 & 0.2913 & 0.2900 \\
\hline & & & & fourth & $x$ & $\times$ & $\times$ & $\times$ \\
\hline \multirow{4}{*}{5} & \multirow{4}{*}{ Jul. 12, 1995} & \multirow{4}{*}{$\begin{array}{l}\text { China } \\
\text { borders } \\
\text { Myanmar }\end{array}$} & \multirow{4}{*}{7.3} & first & Feb. to Apr., 1995 & $17.4633 \vee$ & 2.9453 & 0.4366 \\
\hline & & & & second & Apr. to Jun., 1993 & -11.5929 & 2.0308 & 0.2076 \\
\hline & & & & third & Nov. 1993 to Oct. 1994 & $-7.4821 \vee$ & 1.3083 & 0.0861 \\
\hline & & & & fourth & Sep. 1993 to Nov. 1994 & -6.8692 & 1.2641 & 0.0804 \\
\hline \multirow{4}{*}{6} & \multirow{4}{*}{ Feb. 3, 1996} & \multirow{4}{*}{$\begin{array}{l}\text { Yunnan } \\
\text { Lijiang }\end{array}$} & \multirow{4}{*}{7.0} & first & Mar. to May, 1993 & 3.6293 & 0.5641 & 0.4210 \\
\hline & & & & second & Jan. 1993 to Jun. 1995 & 1.4570 & 0.3597 & 0.1712 \\
\hline & & & & third & Jan.1993 to Feb. 1994 & 1.3167 & 0.3154 & 0.1316 \\
\hline & & & & fourth & Nov.1992 to Feb. 1993 & 0.5076 & 0.2164 & 0.0620 \\
\hline \multirow{4}{*}{7} & \multirow{4}{*}{ May. 12, 2008} & & & first & May to Jul., 2006 & -14.1671 & 2.0315 & 0.4986 \\
\hline & & Sichuan & 80 & second & Jan. 2005 to Jan. 2007 & -8.6209 & 1.1660 & 0.1643 \\
\hline & & Wenchuan & 8.0 & third & Feb. to Apr., 2008 & $6.4793 \vee$ & 1.0013 & 0.1211 \\
\hline & & & & fourth & Apr. to Jun., 2007 & 1.5711 & 0.7851 & 0.0745 \\
\hline & & & & first & May to Jun., 2006 & -14.4247 & 1.8734 & 0.6577 \\
\hline 8 & $\Delta=0$ & Qinghai & 71 & second & Feb. to Apr., 2010 & $9.7744 \vee$ & 0.7497 & 0.1052 \\
\hline & & & & first & Feb. to Mar., 2013 & $11.7708 \vee$ & 1.3844 & 0.4441 \\
\hline & & Sichuan & & second & Jan. to Mar., 2012 & -7.6751 & 1.2888 & 0.3849 \\
\hline 9 & Apr. 20, 2013 & Lushan & 7.0 & third & Oct. to Dec., 2012 & $-3.1351 \vee$ & 0.4654 & 0.0502 \\
\hline & & & & fourth & Feb. to Sep., 2012 & 2.0213 & 0.4085 & 0.0387 \\
\hline
\end{tabular}




\begin{tabular}{|c|c|c|c|c|c|c|c|c|}
\hline $\begin{array}{l}\text { No. } \\
\text { r }\end{array}$ & $\begin{array}{l}\text { Earthquake } \\
\text { date }\end{array}$ & $\begin{array}{l}\text { Earthquak } \\
\text { e location }\end{array}$ & $\begin{array}{l}\text { Earthquake } \\
\text { magnitude (Ms) }\end{array}$ & $\begin{array}{l}\text { The first k } \\
\text { time factor } T_{k}\end{array}$ & $\begin{array}{l}\text { Abnormal occurrence } \\
\text { time }\end{array}$ & $\begin{array}{l}\text { Maximum } \\
\text { anomalous } \\
\text { amplitude } \Delta T\end{array}$ & $\begin{array}{l}\text { The time } \\
\text { factor Mean } \\
\text { square error }\end{array}$ & $\begin{array}{l}\text { abnormal } \\
\text { strain field } \\
\text { ratio }\end{array}$ \\
\hline \multirow{4}{*}{10} & \multirow{4}{*}{ Aug. 8, 2017} & \multirow{4}{*}{$\begin{array}{l}\text { Sichuan } \\
\text { Jiuzaigou }\end{array}$} & \multirow{4}{*}{7.0} & first & Jan. to Mar., 2014 & 1.3225 & 0.4266 & 0.1906 \\
\hline & & & & second & Oct. to Dec., 2015 & -2.5068 & 0.4107 & 0.1766 \\
\hline & & & & third & May to Jul., 2013 & -2.5522 & 0.3883 & 0.1579 \\
\hline & & & & fourth & $\times$ & $\times$ & $\times$ & $\times$ \\
\hline
\end{tabular}

Note: " $\vee$ " said have short imminent anomalies, "x" is no exception or exception is not obvious.
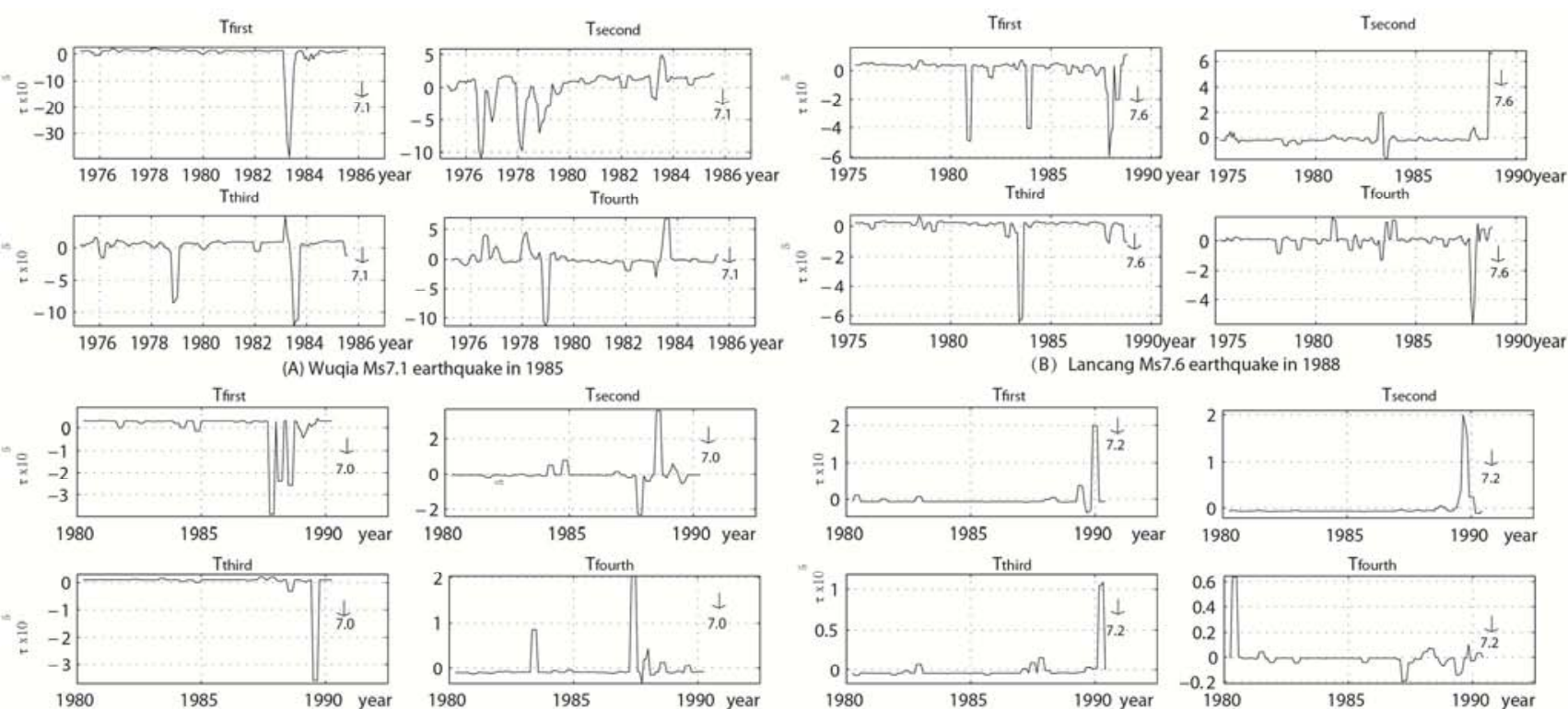

(C) Gonghe Ms7.0 earthquake in 1990
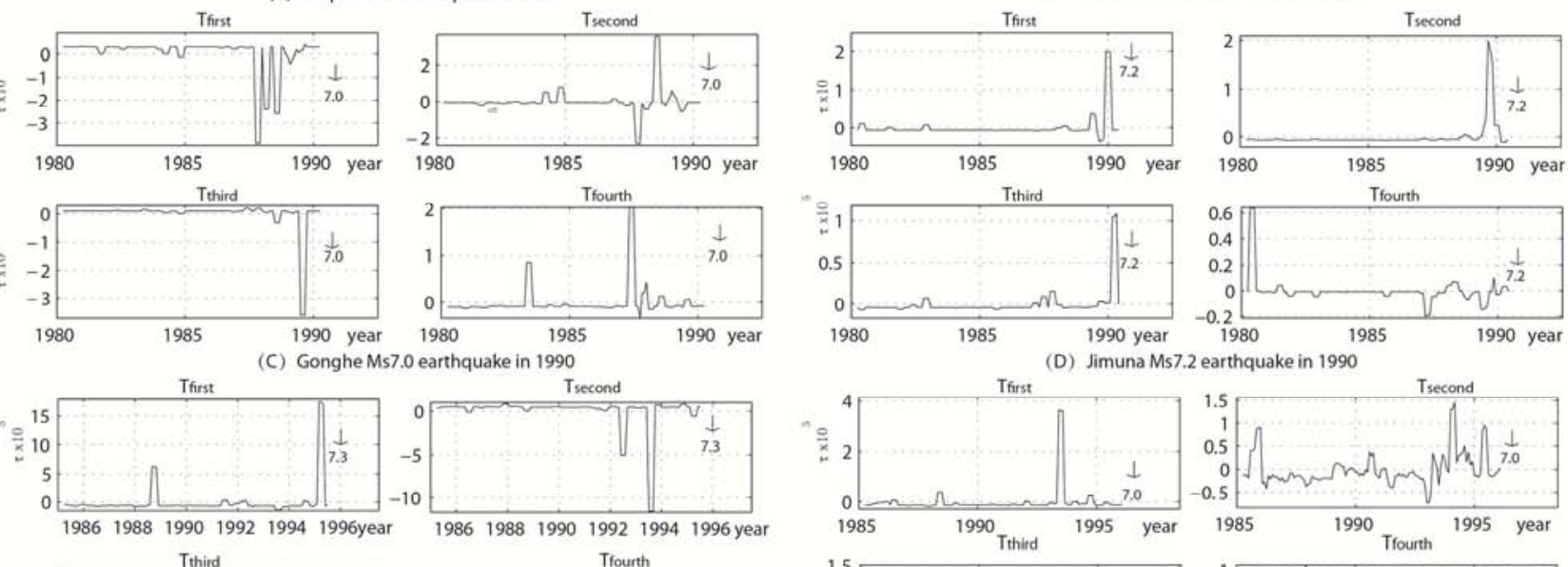

(D) Jimuna Ms7.2 earthquake in 1990
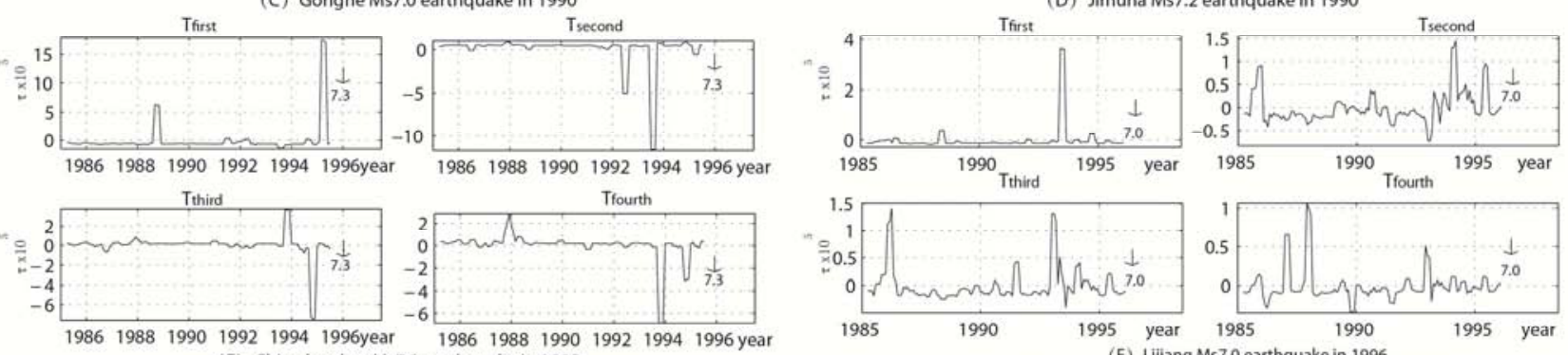
Tfirst ${ }^{(E)}$ China borders Ms7.3 earthquake in 1995 Tsecond
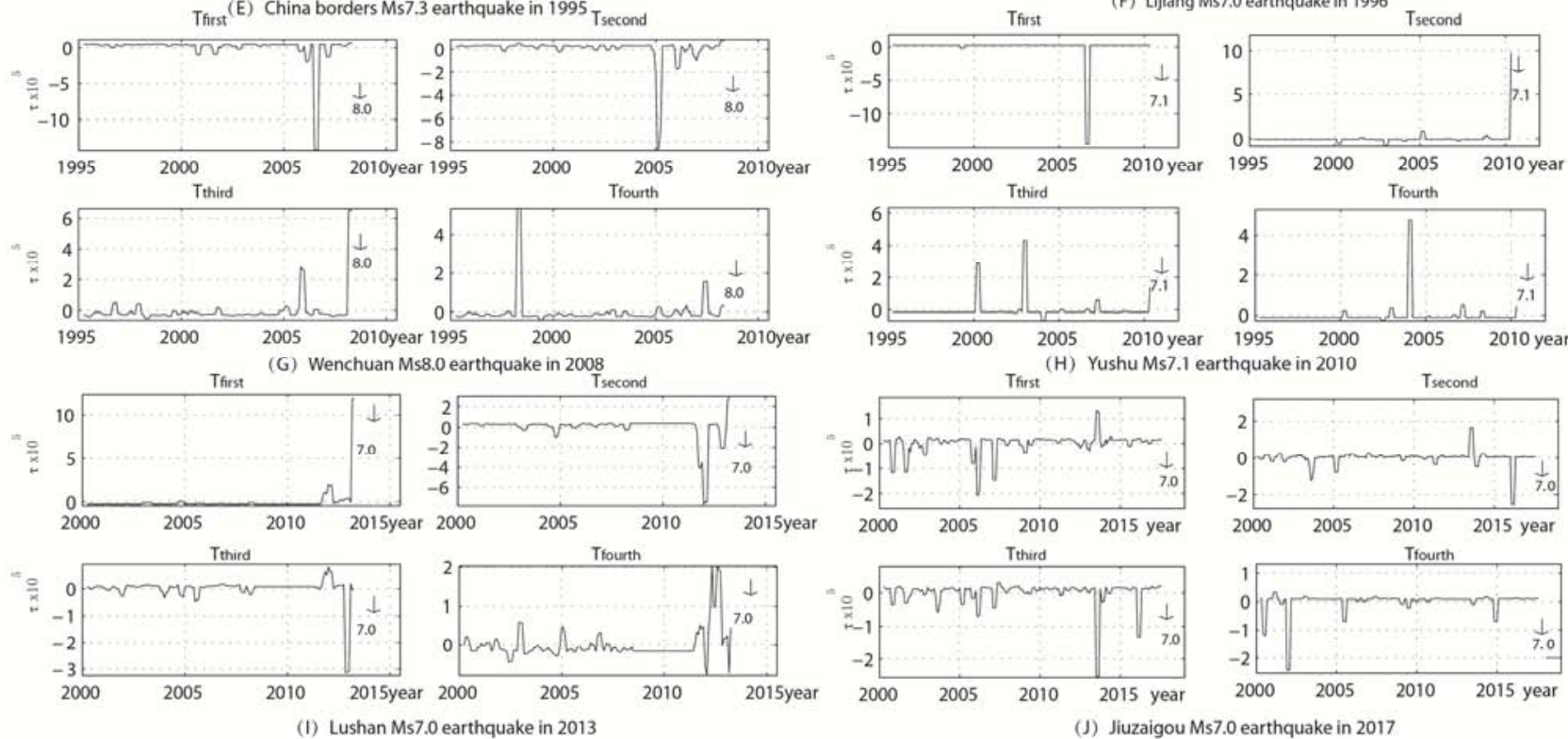

Figure 1. First four typical time factor curves of strain field of ten earthquakes above Ms 7 . 


\subsection{Seismic Parameters and Calculation Precision}

In this paper, the first four eigenvalues and the fitting accuracy of the corresponding typical field are listed in table 1 The time factor of the strain field in each earthquake region shows the change of sudden jump before the earthquake (table 2 ), and the change of time factor of the earthquake strain field (figure 1).

\section{Summary and Discussion}

\subsection{Time Factor Anomaly and Characteristic of Earthquake Strain Field}

As can be seen from figure 1, the time-factor anomaly of seismic strain field is characterized by the following characteristics

(1) Before the magnitude $M \mathrm{~s} 7$ earthquake, the time factor showed a sudden jump or a sudden drop in the background of the seismic strain field.

(2) The magnitude $M \mathrm{~s} 7$ earthquake before the former four typical strain field at least three typical abnormal changes, the vast majority concentrated in the first four typical field, the more weight showed abnormal characteristics show that abnormal component, the more abnormal reliability is higher, and the typical field must be anomalies.

(3) The earliest time of abnormal occurrence is about 3 years before the earthquake, and some of the time factors before the earthquake have obvious characteristics of short-term abnormal changes.

To explain, according to the principle of the method described above formula (3), (4) orthogonal conditions, between various typical field anomalies were independent of each other, so the component shows all the changes are independent of each other exception information.

In fact, before an earthquake of magnitude $M \mathrm{~s} 7$ earthquake strain field time factor appeared significant abnormal changes of kick that could have a significant seismic activity, have a plenty of small earthquakes, some regional swarm activity, also some short-term earthquake activity enhancement, also some in seismic activity in the background of normal longer calm, anomaly characteristics and so on. If they can be identified before a major earthquake, there is no doubt that earthquake prediction will be important. When there is no earthquake, the longer time span curve, may also have some kick point distribution, caused by the sudden jump point and area larger (earthquake magnitude $M_{\mathrm{L}} 4 \sim 5$ ) seismic activity. In general, these jump points only reflect a large change in the individual strain field, which does not have the characteristics of multi-component display abnormality, which can eliminate the time anomaly of the strain field.

\subsection{Comparison of Strain Field and Energy Field}

Table 3 is 2008 Wenchuan Ms8.0 earthquake in Sichuan province of China, 1988 Lancang Ms7.6 earthquake in Yunnan province and 1990 Gonghe Ms7.0 earthquake in Qinghai province, Three times the area of natural orthogonal function expansion of the strain field and before four typical field of comparison, fitting precision table shows four strain field before fitting accuracy is significantly lower than the energy field.

Based on November 6, 1988 Lancang Ms7.6 magnitude earthquake in Yunnan province as an example, analyzing the magnitude Ms7.6 earthquake before the time factor four typical energy field, the four typical energy field has obvious anomalies, abnormal typical field is the total energy ratio is 0.9865 , and the strain field, four typical field before fitting precision is 0.6800 , the first four strain field also has the time factor is unusual, the top two strain field in short imminent anomalies before the earthquake also appear. The typical field time factor curve of the energy field and strain field was analyzed. The results showed that the energy field was the 5th to 8th typical field of the total energy field 0.0895 , There was also an obvious time anomaly before the Chinese Lancang Ms7.6 earthquake (figure 2); And $5 \sim 8$ th strain field is the total strain ratio of 0.1998 , only the eighth time factors of strain field in August 1985 to October values appear to changes in the range of 1.9340 and other typical earthquakes basically see no abnormal change before (figure 3), earthquake examples and many of the same results.

It is found that the strain field has many unique morning advantages by extracting time factor anomaly, comparing strain field and energy field. Firstly, strain analysis is applied to the same earthquake and source parameters. The strain value is much smaller than the energy value. In the orthogonal transformation, the strain field converges slowly, and the anomaly is concentrated in the typical field. It is found that the abnormal variation is mainly concentrated in the first four typical strain fields, and the abnormal energy field is more likely to be distributed. Secondly, the time factor curve of strain field is significantly smaller than that of energy field curve, and it is easy to find the abnormal change of seismic activity in a certain period of time. Finally, the strain field extraction is more reliable and more comprehensive, and it is easy to find out the seismicity generated by the anomaly, and to further eliminate some other disturbance changes in the strain field.

Table 3. The comparison of fitting accuracy between energy fields and strain fields.

\begin{tabular}{|c|c|c|}
\hline Earthquake & $\begin{array}{l}\text { Four typical field fitting accuracy of } \\
\text { energy field method }\end{array}$ & $\begin{array}{l}\text { Four typical field fitting accuracy of } \\
\text { strain field method }\end{array}$ \\
\hline May 12, 2008 the Chinese Wenchuan $M$ s8.0 earthquake & 0.9996 & 0.9020 \\
\hline November 6, 1988, the Chinese Lancang Ms7.6 earthquake & 0.9865 & 0.6800 \\
\hline April 26, 1990, the Chinese Gonghe $M$ s 7.0 earthquake & 0.9997 & 0.9227 \\
\hline
\end{tabular}



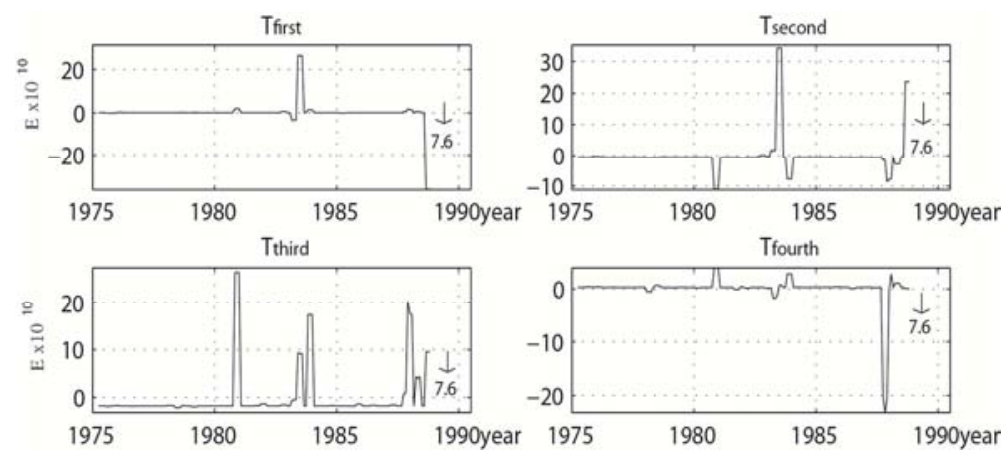

Figure 2. First to fourth typical fields time factors curve of energy field of Yunnan Lancang 7.6 earthquake in 1988.
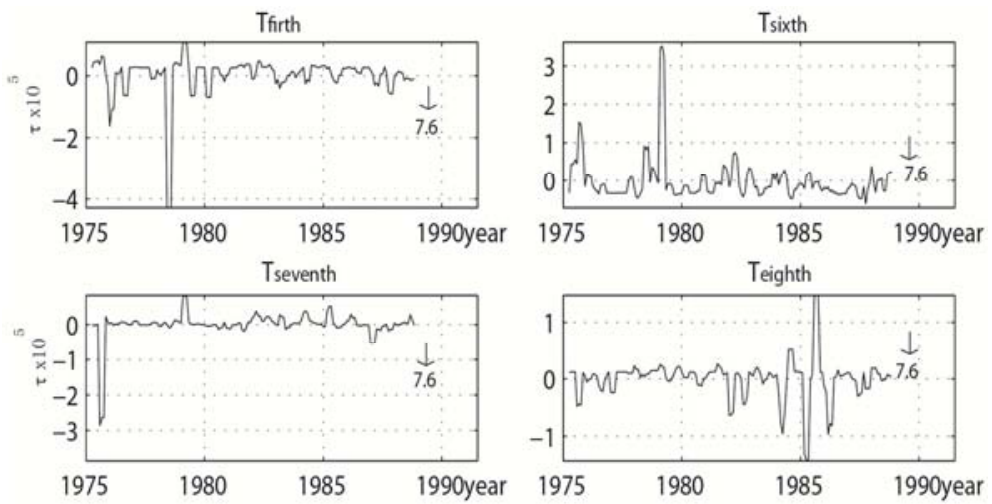

Figure 3. Fifth to eighth typical field time factor curve of strain field of Yunnan Lancang 7.6 earthquake in 1988.

\subsection{Study the Spatial and Temporal Scale Selection}

In principle, seismic activity does not have a large number of repetitions under the same conditions, therefore, strictly speaking, the seismic strain field can be called stochastic function theory is conditional. In the theory of stochastic functions, the conservation of field distribution law is the same external condition, which is to satisfy the uniform condition and the stability condition. However, in practice, these distribution laws are usually unknown, and the statistical time and space scale are generally chosen according to the experience or existing research results. For example, according to the seismic activity in each study area, take 3 months as the time section length, namely, to release the strain value in 3 months instead of the instantaneous value of the field at time point. Ma [10] summarized nine earthquake examples of the earthquake in China, "observed before the flame in two or three years several seismicity anomaly, seismic activity is approaching near-field" wait for a characteristic, two or three years before the earthquake could be a seismic active period. At the same time, considering the stability condition of seismic activity, the study time window is usually selected $10 \sim 15$ years before the earthquake.

The selection of spatial statistical scale of seismic activity can be divided into two regions. One is the seismicity area related to the large earthquake, namely the selected statistical range, and the second is the small scale statistical area, namely the grid. Statistical structures of different scales may be associated with seismic structures of different scales. Mei [11] study of the north China plain, such as 3 times before an earthquake of magnitude $M$ s7 earthquake activity, think that seismic activity area of about $400 \sim 500 \mathrm{~km}$ long axis, and with the development of evolution and the distribution of earthquake activity area area gradually narrowed, 10 years before the large earthquake seismic area is about the range of 3 to 4 arc. In addition, the authors also calculated that the length axis of the 20 magnitude Ms6 earthquake in north China was about $180 \sim 390 \mathrm{~km}$. Therefore, in the analysis of natural orthogonal function expansion, usually around the epicenter around to choose the range of the longitude and latitude about 3 arc as study area, and according to the distribution of earthquake magnitude, the structure and seismic activity, the scope of the study area and make appropriate adjustments. The selected area generally contains the distribution area of the two stages of seismic activity enhancement and attenuation before the major earthquake.

In order to obtain the field function matrix, the equispaced meshes of the region are divided. The grid size should be able to reflect the regional seismic strain distribution characteristics. If the grid is too dense, the seismic distribution types of typical field will be fragmented, and the main characteristics of the field can't be highlighted. At the same time, the covariance matrix tends to deteriorate and the convergence becomes slower. If the grid is divided too thin, it will not be able to reflect the spatial characteristics of seismic activity so that the spatial difference of seismic activity will be weakened, and some important abnormal information may be lost. It is generally convenient to calculate the square grid of 0.5 arc of latitude by $0.5 \mathrm{arc}$, and the rectangular mesh element can be used according to the specific situation. 


\subsection{Study the Stability of Time and Space}

The above table 1 shows that the Wenchuan Ms8.0 earthquake strain field period is from January 1995 to March 2008, and the Lushan earthquake of magnitude $M$ s7.0 the time period from January 2000 to March 2008, the two overlapping period is from January 2000 to March 2008, the figure 1 shows that before the Lushan earthquake of magnitude $M$ s7.0 typical strain curve of time factor in both overlap period (2008 years ago), there is no time factor anomaly. The main reasons for this analysis are as follows:

First, from the table 1 shows the Wenchuan $M \mathrm{~s} 8.0$ earthquake research in the range of (N30.0 to 33.5 arc and E101.5 to106.0 arc), and the Lushan Ms7.0 earthquake research in the range of (N29.5 to 31.5 arc and E29.5 to 31.5 arc), the research scope is different, part of the overlap. In order to compare both the strain energy of time factor differences, to include the scope of both north latitude 29.0 to 33.5 arc, east longitude 101.5 to 106.5 arc as study area, analysis of the typical value distribution of equivalent strain field space is larger, can find the typical strain field time factor differences. According to the author's previous research, the definition of the contour line (or absolute) is defined as the abnormal area in the region greater than 0.05 times 10 to the fifth power (figure 4). By figure 4a known before Wenchuan Ms8.0 earthquake (from January 2000 to April 2008) time factor anomalies are mainly concentrated in a typical and strain (N32.5 arc, E102.0 arc) and (N33.0 arc, E105.0 arc) around these two areas, and the two abnormal area just to be outside the scope of Lushan Ms7.0 earthquake research. And before Lushan earthquake of magnitude $M \mathrm{~s} 7.0$ (from January 2009 to April 2013) typical strain field time factor anomalies are mainly concentrated in the area (N30 to 30.5 arc, E101.5 to $30.5 \mathrm{arc}$ ), for both overlap areas (figure $4 \mathrm{~b}$ ), but the abnormal period do not overlap again, therefore, the analysis of abnormal contour spatial difference, time difference, it before Lushan earthquake of magnitude Ms7.0, time factor in 2008 years ago there was no significant abnormal reason (table 3 ).
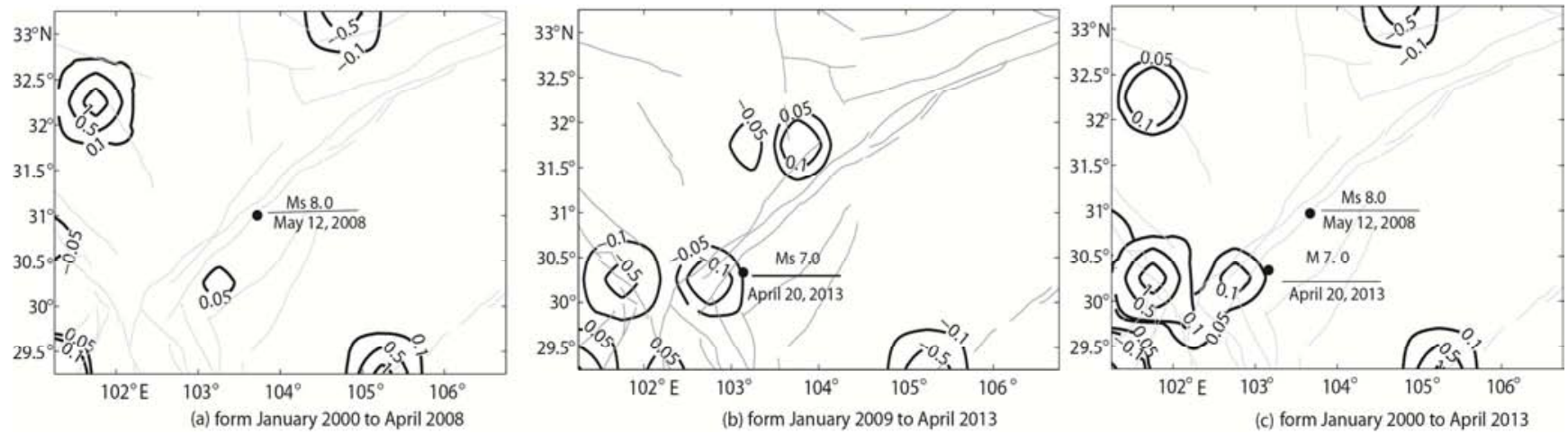

Figure 4. Mean value contour map of strain field in study area (unit: $\left.\times 10^{5}\right)$.

Second, the table 1 and figure 1 shows that before Wenchuan $M$ s8.0 earthquake and Lushan Ms7.0 earthquake the time factor of strain field of the study area is different, and different parts of the typical abnormal strain field time factor is stable? Because of the limited space, only the Wenchuan Ms8.0 earthquake study area (N30.0 to 33.5 arc , E101.5 to 106.0 arc), analyze the stability of the time factor typical strain field.

According to above research methods, to 0.5 arc multiplied by 0.5 arc square grid, calculate the Wenchuan Ms8.0 earthquake research in the typical strain fields of the grid, then compare the space of figure 4 contour abnormalities, found in 2008 Wenchuan Ms8.0 earthquake anomalies are mainly concentrated in front of the typical space distribution, thick line in figure 5 squares as no. 1 , no. 5 and no. 49 grid (named grid number is from down to up from left to right). The center of the three grid coordinates are w1: (N 30.75 arc, E $101.5 \mathrm{arc})$, w5 (N 32.25 arc, E 32.25 arc) and w49 (N 33.25 arc, E 104.75 arc). Study the three earthquake activity in a grid, can be found before Wenchuan Ms8.0 earthquake foreshock activity information, further illustrate the natural orthogonal expansion method can highlight the foreshocks seismicity anomaly of flame front.

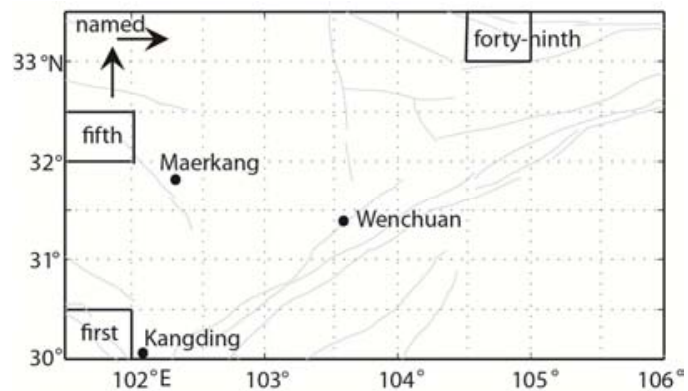

Figure 5. Grid distribution of anomalous before the Wenchuan Ms8 earthquake in 2008 .

Analyze the abnormal grid energy matrix, and reconstitute the energy matrix of the three grid energy values, such as Ei49, Ei5and Ei1, and the energy matrix, which is expressed as:

$$
E=\left[\begin{array}{lll}
E_{1,49} & E_{1,5} & E_{1,1} \\
E_{2,49} & E_{2,5} & E_{2,1} \\
\cdots \cdots \cdots \cdots \cdots \cdots \cdots \cdots . . . \\
E_{m, 49} & E_{m, 5} & E_{m, 1}
\end{array}\right]
$$


Used the natural orthogonal expansion to solve the new energy matrix, get energy sequence curve is shown in figure 6, the figure 6 and Wenchuan Ms8.0 earthquake compared typical strain field time factor graph 1 , can be found that the time factor of three grid energy field changes with the Wenchuan $M \mathrm{~s} 8.0$ earthquake before the time factor three typical strain field at the same time, is 49,5 and 1 grid energy change is composed of three typical strain field of time before the study area factor is the direct cause of the change of the exception. It is further explained that the anomalous principal of the strain field time factor before the Wenchuan Ms8.0 earthquake is stable and reliable.

Third, according to the first two, the analysis of the Wenchuan Ms8.0 earthquake in front of the seismic data, found that 5 grid on January 5, 2005 in the northwest of Maerkang $M_{\mathrm{L}} 5.0$ magnitude earthquake, the earthquake can be seen as early foreshock activity. Then on June 21, 2006 in 49, Wenxian county, Gansu province $M_{\mathrm{L}} 5.4$ earthquake occurs in the grid, from the earthquake happened in Wenchuan earthquake before about one and a half years, can be regarded as the early foreshock activity of the Wenchuan earthquake, medium-term anomaly index significance. Until about two and a half months before the earthquake, 1 February 27, 2008 happened in grid Kangding $M_{\mathrm{L}} 5.0$ earthquake, the earthquake may be regarded as in the Wenchuan earthquake short foreshock activity (table 4), and other form in Wenchuan $M$ s8.0 earthquake had no significant moderate earthquake activity. Similarly with grid method, analysis of Lushan earthquake $M \mathrm{~s} 7.0$ isoline space anomalies (figure $4 \mathrm{~b}$ ), and the combination of table 2 forth four typical strain field before an earthquake of magnitude $M \mathrm{~s} 7.0$ factor is abnormal in the short period of time, can find forth within different grid before earthquake foreshock activity.

It can be seen from the above 3 points that the strain field time factor anomaly before the $M \mathrm{~s} 7.0$ magnitude earthquake in Lushan county was not significantly abnormal before 2008, which is the anomalous spatial distribution in different research areas. However, different regional strain energy time factor anomalies are different, but each regional anomaly is stable and reliable. In addition, before the Wenchuan Ms8.0 earthquake foreshock activity distribution in the peripheral area of the three grid, away from the epicenter of the quake, while no longer near the epicenter, so hard to find abnormal changes of seismic activity near the epicenter, this could be the Wenchuan Ms8.0 earthquake before the anomalous seismic activities found no apparent reason. Normally, for such as Sichuan earthquake zone, $4 \sim 5$ earthquakes is a common earthquake event, before the earthquake is not easy to judge whether a foreshock, but if can combine strain field are likely to find or identify foreshock activity.
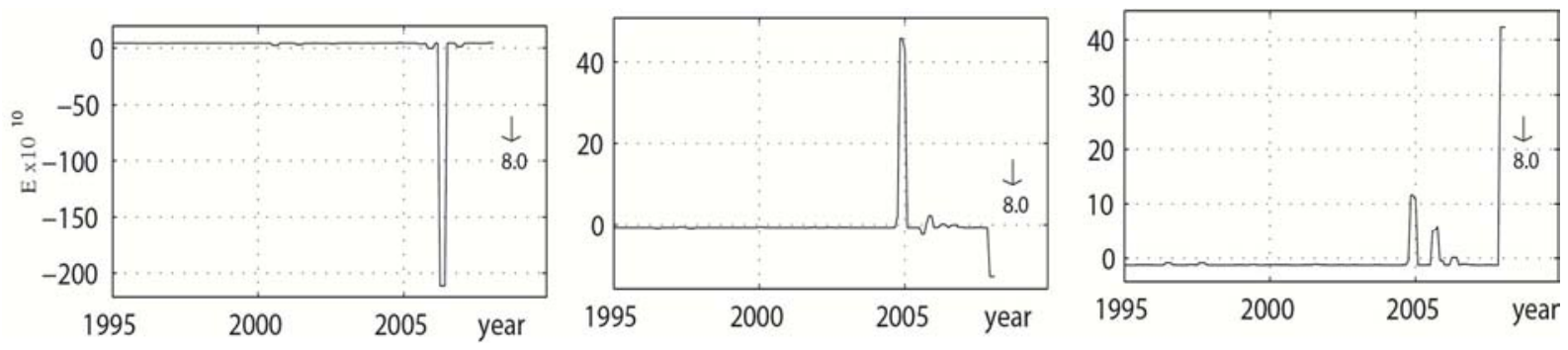

Figure 6. Time series curves of seismic energy anomalies in forty-ninth, fifth and first grids.

Table 4. Foreshock activity of Wenchuan Ms8 and Lushan Ms 7 causing seismic anomalies.

\begin{tabular}{|c|c|c|c|c|c|c|c|}
\hline Earthquake & $\begin{array}{l}\text { The date of the } \\
\text { foreshock }\end{array}$ & Epicenter/ (arc) & $\begin{array}{l}\text { Magnitude } \\
/\left(M_{\mathrm{L}}\right)\end{array}$ & $\begin{array}{l}\text { Earthquake } \\
\text { location }\end{array}$ & Typical field & $\begin{array}{l}\text { Grid } \\
\text { number }\end{array}$ & $\begin{array}{l}\text { Distance from } \\
\text { main shock/ } \\
(\mathrm{km})\end{array}$ \\
\hline Wenchuan & Dec. 15,2004 & E $32.3, \mathrm{~N} 101.6$ & 4.3 & Maerkang & \multirow{8}{*}{$\begin{array}{l}\text { second } \\
\text { second } \\
\text { first, third } \\
\text { third } \\
\text { second, third, fourth } \\
\text { second, third } \\
\text { first, second, third } \\
\text { first, second, third, } \\
\text { fourth }\end{array}$} & fifth & 210 \\
\hline$M \mathrm{~s} 8.0$ & Jan. 5, 2005 & E $32.3, \mathrm{~N} 101.6$ & 5.0 & Maerkang & & fifth & 210 \\
\hline earthquake in & Jun.21, 2006 & E $33.1, \mathrm{~N} 104.9$ & 5.4 & Wenxian & & forty-ninth & 253 \\
\hline 2008 & Feb.27, 2008 & E $30.1, \mathrm{~N} 101.9$ & 5.0 & Kangding & & first & $190 \sqrt{ }$ \\
\hline \multirow{4}{*}{$\begin{array}{l}\text { Lushan } \\
\text { Ms } 7.0 \\
\text { earthquake in } \\
2013\end{array}$} & Oct.19, 2012 & E $30.1, \mathrm{~N} 102.8$ & 4.6 & Tianquan & & second & 24 \\
\hline & Feb.19, 2013 & E $31.2, \mathrm{~N} 105.2$ & 5.3 & Santai & & twenty-fourth & $234 \sqrt{ }$ \\
\hline & Mar.4, 2013 & E $31.0, \mathrm{~N} 103.4$ & 4.1 & Wenchuan & & seventh & $86 \sqrt{ } *$ \\
\hline & Apr.8, 2013 & E $30.8, \mathrm{~N} 103.3$ & 4.2 & chongqing & & eighth & $63 \sqrt{ } *$ \\
\hline
\end{tabular}

Note: " $\sqrt{ }$ " means short impending earthquake; The "*" stands for a controversial pre-quake, which some people believe was an aftershock of the Wenchuan $M$ s 8.0 earthquake, and others believe it was a foreshore of Lushan.

\section{Conclusion and Suggestion}

Based on the research on the abnormal change of time factor of seismic strain field before earthquakes with $M$ s 7 or above in mainland China, this paper gives the following three Suggestions.

Firstly, study the anomaly of time factors before earthquakes above $M \mathrm{~s} 7$, the selection of earthquake catalog data, and generally 10-15 years before the large earthquake seismic area is about the range of 3 to 4 arc.

Secondly, before the $M \mathrm{~s} 7$ earthquake, generally choose the epicenter of the small and medium earthquakes around the magnitude 5 or more of the minimum complete magnitude, it is best not to delete aftershock data. However, if a $M$ s7 or 
above earthquake occurs and the epicenter is within $3 \sim 4$ arcs within 10 years, another $M \mathrm{~s} 7$ or above earthquake occurs, the aftershock of the previous $M \mathrm{~s} 7$ earthquake shall be deleted to prevent the aftershock of the previous $M$ s 7 earthquake.

Thirdly, it is better to select the seismic strain field method rather than the seismic energy method to study the anomalies of time factors before earthquakes of $M \mathrm{~s} 7$ or above, because the seismic strain method has more advantages than the seismic energy method.

\section{Two Key Points:}

i Method highlights. By using the method of natural orthogonal function, the seismic energy field is a unique advantage of the seismic energy field.

ii Research results are highlights. This paper studies the strain field before the magnitude $M \mathrm{~s} 7$ earthquakes in mainland China since 1980, and finds out the new characteristics and causes of the time factor before the earthquake.

\section{References}

[1] CYRI llic, KAI Zha Kai Ei Qi.1971: Random Function Theory and Its Application in Hydrology and Meteorology. Press Seismological (in Chinese Beijing).

[2] MA Kaiy, DING Yuguo, TU Qi.1993: Principles and Methods of Climate Statistics . Press Seismological (in Chinese Beijing).

[3] YANG Mingzhi, ZHAO Weiming.2004: Statistical Analysis on Energy Field of Seismicity in Ningxia and Its Neighborhood Region. Acta Seismologica Sinica, 26(5): 516-522.
[4] LUO Guofu, YANG Mingzhi.2005: Space-time Distributed Characteristics on Energy Field of Earthquake in the Yunnan Region. Earthquake Research in China, 21(3): 13-18.

[5] LUO Guofu, TU Hongwei, MA Heqin. et. al 2011: Analysis on Energy Field of The Seismic Activity in Earthquake Hazard Area From The Northwest to The South of Yunnan. Journal of Seismological Research (in Chinese), 34(4): 285-291.

[6] YANG Mingzhi, MA Heqin.2012: Analysis of Regional Seismic Energy Field before Wenchuan Ms8.0 Earthquake. Progress in Geophysics (in Chinese), 27(3): 0872-0877.

[7] MA Heqin, YANG Mingzhi. 2013: Research on The Energy Field about Yushu $M_{\mathrm{S}} 7.1$ Earthquake in Qinghai in 2010. Journal of Seismological Research (in Chinese) 35(4): 487-490.

[8] YANG Mingzhi, MA Heqin. 2013: Features of Time Factor Anomalies of Regional Energy Field before Large Earthquake. Earthquake (in Chinese), 33(3): 107-115.

[9] LUO Guofu, ZENG Xianwei, MA Heqin. et. al. 2014: Analysis of Energy Field of Seismic Activity before The Minxian-Zhangxian $M_{\mathrm{S}} 6.6$ Earthquake in China . Earthquake Engineering Journal (in Chinese), 36(2): 314-319.

[10] MA Zhongjin, Fu Zhengxiang.1990: Study on Earthquake Prediction in China.//Observation and Physical Basis of Medium Term Earthquake Prediction. Press Seismological (in Chinese Beijing).

[11] MEI Shirong. 1997: General Characteristics of Long Term Evolution of Seismicity before Strong Earthquakes in North China.//Theory and Method of Short Impending Earthquake Prediction. Press Seismological (in Chinese Beijing). 